hep-ph/9712455

FTUV/97-71

IFIC/97-102

FISIST/14-97/CFIF

\title{
Borexino as a test of solar matter density fluctuations *
}

\author{
H. Nunokawa ${ }^{\mathrm{a}}$, A. Rossi ${ }^{\mathrm{b}}$, V. Semikoz ${ }^{a, \mathrm{c}}$ and J.W.F.Valle ${ }^{a}$ \\ ${ }^{a}$ Instituto de Física Corpuscular - C.S.I.C., Departament de Física Teòrica, Universitat de València \\ 46100 Burjassot, València, Spain \\ ${ }^{\mathrm{b}}$ Dept. de Fisica, Inst. Superior Tecnico, 1096 Lisbon Codex, Portugal \\ ${ }^{\mathrm{c}}$ Institute of the Terrestrial Magnetism, the Ionosphere and Radio Wave Propagation of the Russian \\ Academy of Sciences, IZMIRAN, Troitsk, Moscow region, 142092, Russia
}

This talk summarizes some results of our recent work focusing on the possibility to test solar matter density fluctuations by the future Borexino experiment.

\section{Introduction}

The solar neutrino deficit observed in Homestake, SAGE, GALLEX and Kamiokande experiments [1] has been confirmed by the new result from Super-Kamiokande experiment [2]. The most successful explanation of the deficit is given by the resonant flavor conversion of neutrinos (MSW mechanism) [3] in the solar interior.

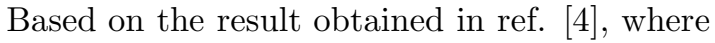
we have studied the impact of random density perturbation on the MSW solution to the solar neutrino problem, this talk features the possibility to test such fluctuations by the future Borexino experiment. The effect of density perturbations on the neutrino propagation has also been studied in refs. 5 - 9 .

\section{Basic assumptions and general features of the effect}

In general, the matter density in the solar interior may fluctuate around its mean values as,

$\rho=\langle\rho\rangle+\delta \rho$,

where the symbol $\langle\ldots\rangle$ means to take average. We assume that the perturbation is random and have a Gaussian distribution according to the following correlation function:

$$
\left\langle\delta \rho(r) \rho\left(r^{\prime}\right)\right\rangle \sim \xi^{2}\langle\rho\rangle^{2} L_{0} \delta\left(r-r^{\prime}\right)
$$

where $\xi \equiv \delta \rho / \rho$ is the magnitude of the fluctuation, $L_{0}$ is the correlation length, which corre-

\footnotetext{
*Talk given by H. Nunokawa in Taup97, Gran Sasso, Italy,
} September, 1997 sponds to the scale of the fluctuation.

Here we only give the basic idea of how we can compute the survival probabilities of neutrinos in the presence of fluctuations and refer the reader to ref. [4] for detail. We start from the system of usual two-flavor evolution equations for neutrinos in matter but with the fluctuation term in eq. (1). The system of equations is averaged over the fluctuation ensemble, taking into account the relation (2), before computing the final probability. In this way the evolution equations for the averaged survival probability $\langle P\rangle$ already include the effect of the fluctuation. An alternative approach, taking the ensemble average of $P$ after solving the equation for many different profiles of $\delta \rho$ is considered in ref. [9].

An important assumption in our analysis is

$l_{\text {free }} \ll L_{0} \ll \lambda_{m}$,

where $l_{\text {free }} \sim 10 \mathrm{~cm}$ is the mean free path of the electrons in the solar medium and $\lambda_{m}$ is the neutrino oscillation length in matter. We show the effect of density fluctuation on the MSW mechanism in Fig. 1. As we can see from the figure the general feature of the effect is to suppress the MSW mechanism. Although the effect seems to be rather large, the two MSW solutions (large and small mixing angle) are found to be rather stable [4], which implies that the current solar neutrino experiments are not very sensitive to the density fluctuations. However, the upcoming Borexino experiment [11], aiming to detect ${ }^{7} \mathrm{Be}$ neutrinos, could 'resolve' this effect. In order to see that these neutrinos are the ones most affected by the 
fluctuation we have indicated in Fig. 1 by the vertical lines the position where ${ }^{7} \mathrm{Be}$ neutrinos fall in for $\delta m^{2} \sim 10^{-5} \mathrm{eV}^{2}$.
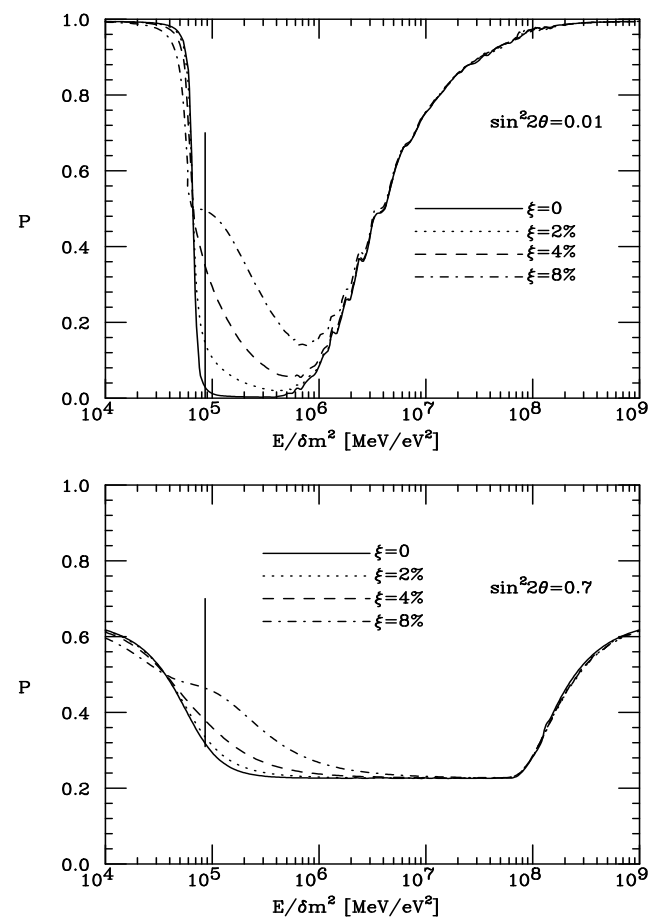

Figure 1. The averaged solar neutrino survival probability as a function of $E / \delta m^{2}$, which is obtained by numerical integration (imposing $L_{0}=$ $0.1 \times \lambda_{m}$ ), is plotted for $\sin ^{2} 2 \theta=0.01$ (upper panel) and 0.7 (lower panel). We have used the density profile in the Sun from ref. [10].

\section{Effect on Borexino Experiment}

Let us see more qualitatively the effect of fluctuation on Borexino experiment. In Fig. 2 we plot the iso $Z_{B_{e}} \equiv R_{B_{e}}^{M S W} / R_{B_{e}}^{B P 95}$ contours in the Borexino detector for the $\nu_{e} \rightarrow \nu_{\mu, \tau}$ conversion without and with density fluctuations. Here $R_{B_{e}}^{M S W}$ and $R_{B_{e}}^{B P 95}$ refer to the expected event rate with and without MSW mechanism.

In the following we assume that the standard solar model prediction is correct and the solar neutrino deficit is explained by small mixing angle MSW solution, either due to $\nu_{e} \rightarrow \nu_{\mu, \tau}$ or $\nu_{e} \rightarrow \nu_{s}$ where $\nu_{s}$ is a sterile neutrino.
For the case without fluctuations the Borexino signal is expected to be in the range $Z_{B e} \sim$ $0.2 \div 0.7$ (see the upper panel in Fig. 2) whereas in the presence of the fluctuation $\xi=4 \%$ (see the lower panel in Fig. 2), the minimal allowed value for $Z_{B e}$ becomes higher, $Z_{B e} \geq 0.4$. Hence, if the MSW mechanism is responsible for the solar neutrino deficit and Borexino experiment detects rather low signal, $Z_{B e} \sim 0.2$ (with good accuracy) this implies that a few \% level of matter fluctuations in the central region of the Sun is unlikely. The same argument can be applied to $\nu_{e} \rightarrow \nu_{s}$ solution in case experiments like Super-Kamiokande and/or SNO should establish it. The expected signal in Borexino is very small $Z_{B e} \approx 0.02$ for $\xi=0$ (see the upper panel in Fig. 3). On the other hand with $\xi=4 \%$, the minimum expected signal is 10 times higher than in the noiseless case, so that if Borexino detects a rate $Z_{B e} \lesssim 0.1$ (see the lower panel in Fig. 3) this would again exclude noise levels above few $\%$.

We note that our discussion is valid only if $L_{0}$ is smaller than $\sim 5 \times 10^{4} \mathrm{~km}$ which is obtained from the condition (3) at the resonance. If $L_{0}$ become larger than this value, our approach is inadequate and moreover, the effect of random perturbations ceases to exist 9].

\section{Conclusion}

We have discussed the possibility to test the effect of density fluctuation in the Sun by Borexino experiment. We conclude that if the small angle MSW solution is established and if we see rather small signal in Borexino this implies the exclusion of the density fluctuation at the level of a few \%, which could be compared with independent informations from Helioseismology.

We finally note that ref. [9] concludes that no sizable effect from the density fluctuation on the MSW mechanism can be expected if the fluctuation is in the form of Helioseismic waves. We, however, feel that it is too early to conclude that the random perturbations at a few $\%$ level with the correlation length we considered here is completely unlikely.

More detailed discussion on this work is found in ref. 肺. 

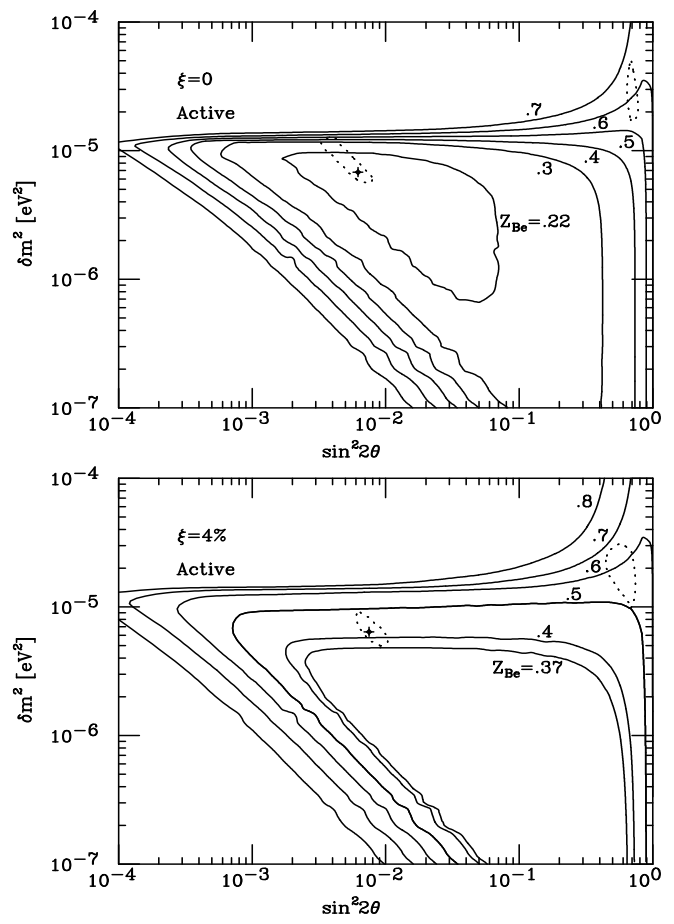

Figure 2. The iso contours of $Z_{B_{e}}$ (see the text) in the Borexino detector for the $\nu_{e} \rightarrow \nu_{\mu, \tau}$ conversion are plotted. Upper panel refers to the case with no fluctuation, $\xi=0$ whereas the lower panel refers to the case with $\xi=4 \%$. The region allowed at $90 \%$ C.L. is also plotted by dotted lines.

\section{REFERENCES}

1. B. T. Cleveland et al., Nucl. Phys. B (Proc. Suppl.) 38 (1995) 47; K. S. Hirata et al. Phys. Rev. Lett. 20 (1990) 1297; P. Anselmann et al., Phys. Lett. B 327 (1994) 377; J. N. Abdurashitov et al., Phys. Lett. 328 (1994) 234.

2. Y. Totsuka, for Super-Kamiokande Collaboration, talk given at the Lepton-Photon '97, August 1997, Hamburg, Germany.

3. S. P. Mikheyev and A. Yu. Smirnov, Sov. J. Nucl. Phys. 42 (1986) 913; Sov. Phys. Usp. 30 (1987) 759; L. Wolfenstein, Phys. Rev D17 (1978) 2369; ibid D20 (1979) 2634.

4. H. Nunokawa, A. Rossi, V. Semikoz and J.W.F.Valle, Nucl. Phys. B 472 (1996) 495.

5. A. Schafer and S. E. Koonin, Phys. Lett. B185 (1987) 417; P. I. Krastev and A. Yu Smirnov, Phys. Lett. B226 (1989) 341; Mod.
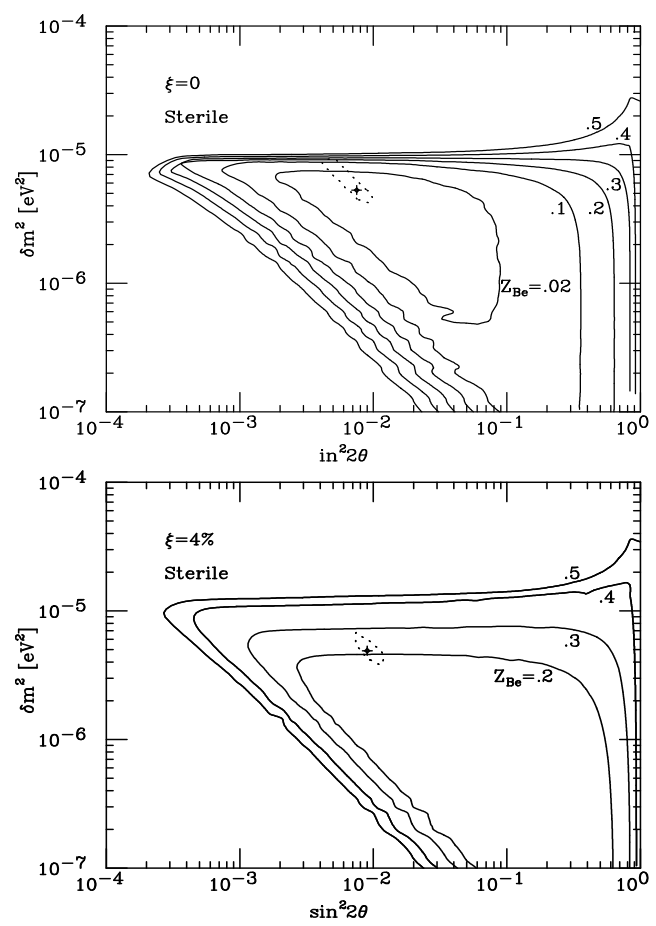

Figure 3. Same as in Fig. 2 but for the case of $\nu_{e} \rightarrow \nu_{s}$ conversion where $\nu_{s}$ is a sterile state.

Phys. Lett. A6 (1991) 1001; R. F. Sawyer, Phys. Rev. D42 (1990) 3908; A. Abada and S.T. Petcov, Phys. Lett. B279 (1992) 153.

6. F. N. Loreti and A. B. Balantekin, Phys. Rev. D50 (1994) 4762; A. B. Balantekin, J.M. Fetter and F.N. Loreti, Phys. Rev. D54 (1996) 3941; F. N. Loreti et al., Phys. Rev. D52 (1996) 6664.

7. E. Torrente-Lujan, hep-ph/9602398.

8. C.P. Burgess and D. Michaud, Ann. Phys. (NY) 256 (1997) 1.

9. P. Bamert, C.P. Burgess and D. Michaud, hep-ph/9707542, to appear in Phys. Rev. D; P. Bamert, hep-ph/9708224; C.P. Burgess, hep-ph/9711425.

10. J. N. Bahcall and M. H. Pinsonneault, Rev. Mod. Phys. 67 (1995) 781.

11. C. Arpesella at al., BOREXINO Collaboration, Proposal of BOREXINO (1991). 